\title{
Konversion in einer \\ europäischen Stadt
}

\section{Ein Mannheimer Projekt setzt Maßstäbe der Bürgerbeteiligung}

PETER KURZ

Dr. Peter Kurz ist Oberbürgermeister der Stadt Mannheim.

www.mannheim.de

In vielen deutschen Städten wurden nach der

Wiedervereinigung durch den Abzug ausländischer

Streitkräfte große ehemals militärisch genutzte

Flächen frei. In Mannheim ist dies die "US-Garnison

Mannheim « mit etwa 2.000 Gebäude mit 2.200

Wohnungen auf einer Fläche von etwa 500 Hektar.

Die Stadt Mannheim hat zu der Frage, wie diese

Flächen und Bauten künftig genutzt werden sollen, eine umfangreiche Bürgerbeteiligung in Gang gesetzt.

Eine »europäische Stadt" ist - im globalen Vergleich - eine, in der um ihren Zusammenhalt, ihre Vielfalt und die Bedingungen ihrer Produktivität miteinander gerungen wird in kommunaler Selbstverwaltung, sozusagen auf dem Marktplatz der ursprünglichen Athener Demokratie. der handelnden Akteure in der Kommunalpolitik. Denn diese haben uns beim erregten Protest um »Stuttgart 21« im Kern so irritiert und in Wellen sogar Landes- und Bundespolitik nachdenklich gemacht.

Einer Stadt wie Mannheim durch den Abzug von US-Militär über fünf

\section{"Die Entdeckung der Langsamkeit in der Planung: Unsere Generation muss nicht für alles schon eine dauerhafte Antwort geben wollen«}

Wie kann die europäische Stadt in einer neuen Balance der Selbstverwaltung aus repräsentativ-demokratischen Strukturen und den direkten, demokratischen deliberativen Beteiligungsformen angemessen mit Großprojekten umgehen? Wie können wir Menschen gewinnen, emotional und rational als Bürger und Bürgerinnen teilzuhaben an solchen Prozessen?

Neue Regeln zu entwickeln ist dabei auch ein Bekenntnis zu Gefühlen, Hoffnungen, Ängsten und Sehnsüchten
Millionen Quadratmeter (oder 500 Hektar) Flächen "zurückzugeben " ist kein Verwaltungsvorgang, sondern eine Herausforderung für die Stadtgesellschaft insgesamt. So wie sich Städte auf Industrie- und Klimawandel, auf $\mathrm{Zu}$ wanderung und Alterung nicht isoliert auf Ressort- oder Zielgruppenebene, sondern insgesamt einzustellen haben, ist die Konversion ein Anlass zur neuen Selbstbestimmung der Stadtgesellschaft und Positionierung in der Region, ist Anlass nachzudenken über Verantwor- 
tung gegenüber ihrer eigenen Stadtgeschichte und für ihre nachwachsenden Generationen. Großprojekte sind eine Chance zur Selbstvergewisserung als europäische Städte im beschriebenen Sinn und keine unlösbaren Demokratieprobleme.

Mannheim hat dies mit einem neunmonatigen Prozess der Bürgerbeteiligung getan, der mit der Weichenstellung beginnt, die richtigen Fragen zu stellen - nämlich der Aufforderung nach Ideen, Eigenaktivitäten und Unternehmensmut - und mit der Vorlage eines Weißbuches endet, das der Gemeinderat beschließt. Damit kommt dem Gemeinderat die Aufgabe zu, der Bürgerschaft zuzuhören und deren Gewichtungen in einem Abschlusspapier Beschlusscharakter zu verleihen. Der Rat legt Eckpunkte fest, konzentriert alle Möglichkeiten auf erreichbare Ziele und wertbildende Marken und öffnet die nächste Tür der Bürgerbeteiligung: Mitgestaltung der Themen und Fachaufgaben und das Aufgreifen von "großen « Ideen.

Möglichst alle Gruppen einer Stadtgesellschaft für das Thema Konversion anzusprechen, heißt ihre Einstellungen und Gefühle zu ihrer Stadt aufzunehmen, Projekte wie die Bundesgartenschau, Seen oder interkulturelle und intergenerative Ideen ernsthaft zu verfolgen, die sie stolz sein lässt auf "unsere Stadt « und damit Produktivkräfte mobilisiert, ohne die eine Stadt nicht wachsen könnte. Die Erfahrungen dieses bisher sehr erfolgreichen Prozesses unter Mitwirkung von renommierten externen Experten lehren jetzt schon, in der Planung Qualität vor Quantität zu stellen.

Denn Qualität ist nur durchsetzbar, wenn nicht in allem sofort der Kompromiss gesucht werden muss, weil jede Anmerkung zu einer Investitionsentscheidung, jede kritische Debatte über Qualität als Behinderung und Schwächung des Standorts betrachtet wird. Und manchmal geht es um die Entdeckung der Langsamkeit, was bedeutet, dass nicht unsere Generation für alles schon eine dauerhafte Antwort geben muss und sollte.

Die größte Sorge der Bürgerinnen und Bürger, die sich im Diskussionsprozess der letzten Monate beteiligt haben, ist nicht, dass Politik und Verwaltung ihre Anliegen nicht ernst nehmen, son-

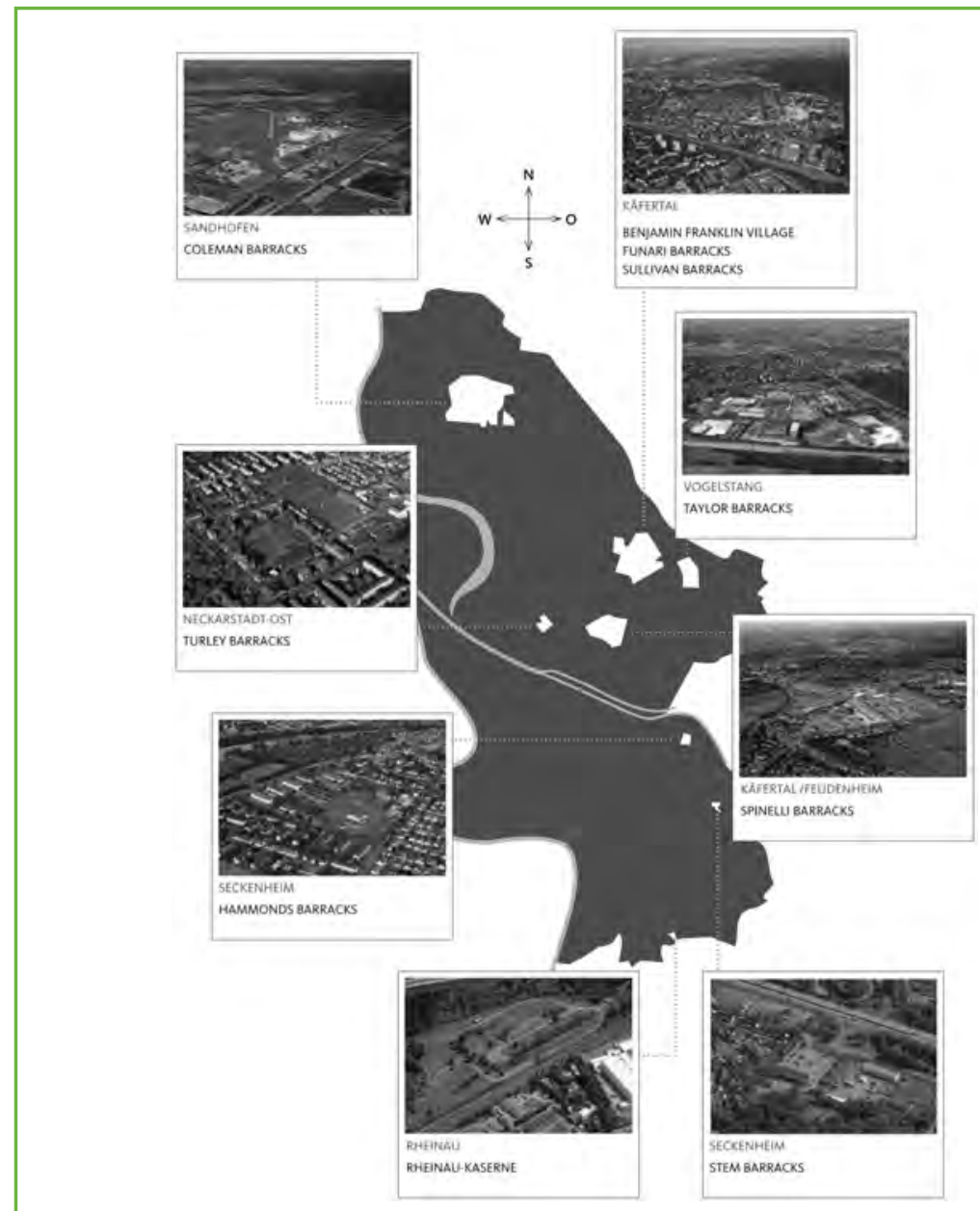

In Mannheim stehen bis zum Jahre 2015 über 500 Hektar bislang militärisch genutzter Flächen für eine städtebauliche Entwicklung zur Verfügung. In einer neunmonatigen ersten Phase der Bürgerbeteiligung wurden in Mannheim zahlreiche Ideen und Vorschläge gesammelt, was mit diesen Gebieten nun geschehen soll. Politiker und Planer haben mit Bürgerinnen und Bürger andere Konversionsprojekte besichtigt, in Workshops diskutiert und thematische Schwerpunkte gesetzt. Dieser Prozess und seine Ergebnisse wurden in einem Weißbuch auf ingesamt 182 Seiten dokumentiert. Das Weißbuch »Offene Räume - Starke Urbanität« steht im Internet zum Herunterladen zur Verfügung.

www.mannheim.de/stadt-gestalten/weissbuch

dern, dass nicht die Kraft besteht, Konzeptionen lange und ausdauernd mit Investoren zu verhandeln und durchzusetzen. Als vermittelnde Instanz haben wir freiwillige und neutrale Zukunftslotsen ausgesucht, die uns bei der Moderation der nächsten Schritte helfen.

Wir haben uns auf einen Weg der Bürgerbeteiligung gemacht, der ungewöhnlich breit angelegt war und dennoch in hohem Tempo zu Wegweisungen geführt hat, zu denen wir in Politik und Verwaltung ohne diese Impulse so nicht gekommen wären.

Die Ernsthaftigkeit der Diskussion der Teilnehmerinnen und Teilnehmer unterschiedlicher Herkunft hat mich sehr beeindruckt. Wir wollen diesen
Weg auch deshalb weiter gehen, weil es nicht nur darauf ankommt, Planungen zu entwickeln, sondern Partner für die Umsetzung zu gewinnen.

Das erste Mannheimer Weißbuch zur Konversion und Bürgerbeteiligung dokumentiert ein Verfahren, das nicht einer Beteiligungsform genügt, sondern einen Prozess dokumentiert, der Verwaltung, Gemeinderat, Bürgerschaft, Interessengruppen ihren Platz zuweist auf einem Feld, das wir modern, aber noch uneingelöst "urban governance " nennen. Es bleibt abzuwarten, ob sich daraus neue Erfahrungen und Spielregeln gewinnen lassen für die Entwicklung und Umsetzung von großen Gestaltungsaufgaben. 